\title{
Tendonitis in the upper extremity: Is it a useful or useless diagnosis?
}

\author{
Arnis Freiberg MD FRCSC FACS
}

$\mathrm{T}$

he term 'tendonitis' has become a popular diagnosis. Can this diagnosis be used for pain in the forearm, in places were there are no tendons? Can a tendon without a sheath become inflamed and cause pain? What is the etiology? An attempt is made to discuss some of these questions.

"Doctor, my regular doctor says that I have tendonitis! What is it? What can you do for me?" Not an uncommon question heard in the hand clinic these days. This diagnosis has become popular not only with general practitioners, but also with some physiatrists and radiologists. We are familiar with various forms of tenosynovitis and accept the fact that tendon sheaths can become inflamed, cause pain or triggering, but what about areas such as the upper forearm where there are no tendons, only muscles? Would a diagnosis of myofascial pain syndrome (another sometimes controversial diagnosis) be more appropriate?

To educate myself, I went to the literature and found some interesting information!

What is the correct spelling? Tendonitis or tendinitis? We spell 'tendon', not 'tendin'... why then, a while ago, Wikipedia said that "tendonitis is the non-medical spelling"?, currently saying that the two terms are interchangeable!

What about some definitions? 'Tendinopathy' has replaced the term 'tendonitis' as a generic description of clinical conditions associated with pain, swelling and impaired performance in and around tendons from overuse. 'Tendinosis' is defined as chronic degeneration of the tendon and can also be related to partial tendon rupture. This term is also used by some radiologists.

\section{Causes and etiology}

What do we know about causes and etiology? It is generally accepted that causes are still unknown and have only been theorized. There is a proposed theory: increased repetitive stress and force on the tendon cause an increased risk of injury. Over time, intrinsic and extrinsic factors result in the development of tendinopathies.

Intrinsic factors are defined as malalignment, muscular imbalance and muscular insufficiency. Extrinsic factors are defined as training errors in sports, poor ergonomics and equipment in industry and others.

\section{Anatomy}

Some relevant factors in tendon anatomy. We know that collagen fibres from within the muscle organ are continuous with those of the tendon. Pain and tenderness over this area would more likely indicate a diagnosis of myofascial pain rather than tendonitis.

Tendon length varies in all muscle groups and from person to person, and is determined by genes. It may explain the fact that some arms are simply not suitable for certain activities, especially on a repeated or prolonged basis.

Internal tendon bulk is thought to contain no nerve fibres, so the question is: what causes pain in tendons without a sheath?

Some thoughts about relevant muscle anatomy and physiology. We humans, much like turkeys, are said to have weak and strong muscles - 'white meat' and 'dark meat'.

Some individuals have more weak muscles than strong and use some of these muscles for activities for which they were not designed. I think that we have to realize this, especially when dealing with work-related difficulties, and advise a change of jobs instead of trying to find physical or surgical solutions. We must also realize that muscle function starts to deteriorate after the age of 40 years. On the cellular level, sarcolemmal bridges may become too short or too long and may be responsible for abnormal muscle function. All of these factors may be responsible for the diagnosis of 'tendonitis'.

\section{Myofascial pain syndrome}

Although I have been reluctant to make the diagnosis of myofascial pain syndrome in the past, I think that this diagnosis is more appropriate than tendonitis, especially in areas where there are no tendons!

Myofascial pain syndrome is a painful musculoskeletal condition, characterized by the development of trigger points. It is a common cause of musculoskeletal pain. The trigger point is usually present in a muscle or at the junction between muscle and fascia. In contrast, the trigger point in tendonitis is at the junction between tendon and its bony insertion, or perhaps, at the muscle origin, such as seen in 'tennis elbow'.

We must also keep in mind that if a patient presents with more than one definite trigger point, the diagnosis may be fibromyalgia, and a referral to a rheumatologist may be appropriate.

\section{Treatment strategies}

What are the current treatment strategies? First and foremost: assess and correct ergonomics in the work place or avocational activities, such as sports or performing arts. Involve and work

University Health Network, Toronto Western Hospital; Department of Surgery, Division of Plastic Surgery, University of Toronto, Toronto, Ontario

Correspondence and reprints: Dr Arnis Freiberg, Toronto Western Hospital, Hand Program 2-East Wing, 399 Bathurst Street, Toronto, Ontario M5T 2S8. Telephone 416-603-5691, fax 416-603-5297, e-mail arnisfreiberg@rogers.com 
with a competent hand therapist. Avoid so commonly prescribed aggressive physiotherapy. Think more of rest than exercise - you are dealing with an inflamed condition or perhaps a partially torn tendon. Use appropriate custom made splints. Consider steroid injections. Surgery is seldom indicated.

In summary, remember that tenosynovitis is more common than tendonitis. Think of myofascial pain syndrome when the trigger point is over the muscle-tendon junction.
Make every effort to make a definite diagnosis and prescribe appropriate and specific treatment. Do not forget that some individuals may not be suited for certain activities, and the time may have come to switch jobs or recreational activities.

And finally, if you are unable to make a definite diagnosis, send a note to the referring physician that you agree with his/her diagnosis of tendonitis, they will understand! 